\title{
CoSMOS: Performance of Kurtosis Algorithm for Radio Frequency Interference Detection and Mitigation
}

\author{
Misra, Sidharth; Kristensen, Steen Savstrup; Skou, Niels; Søbjærg, Sten Schmidl
}

Published in:

IGARSS: 2007 IEEE INTERNATIONAL GEOSCIENCE AND REMOTE SENSING SYMPOSIUM

Link to article, DOI:

10.1109/IGARSS.2007.4423403

Publication date:

2007

Document Version

Publisher's PDF, also known as Version of record

Link back to DTU Orbit

Citation (APA):

Misra, S., Kristensen, S. S., Skou, N., \& Søbjærg, S. S. (2007). CoSMOS: Performance of Kurtosis Algorithm for Radio Frequency Interference Detection and Mitigation. In IGARSS: 2007 IEEE INTERNATIONAL

GEOSCIENCE AND REMOTE SENSING SYMPOSIUM: SENSING AND UNDERSTANDING OUR PLANET (pp. 2714-2717). IEEE. https://doi.org/10.1109/IGARSS.2007.4423403

\section{General rights}

Copyright and moral rights for the publications made accessible in the public portal are retained by the authors and/or other copyright owners and it is a condition of accessing publications that users recognise and abide by the legal requirements associated with these rights.

- Users may download and print one copy of any publication from the public portal for the purpose of private study or research.

- You may not further distribute the material or use it for any profit-making activity or commercial gain

- You may freely distribute the URL identifying the publication in the public portal 


\title{
CoSMOS: Performance of Kurtosis Algorithm for Radio Frequency Interference Detection and Mitigation
}

\author{
Sidharth Misra, Steen. S. Kristensen, Sten. S. Søbjærg and Niels Skou \\ Danish National Space Center - B348 \\ Technical University of Denmark \\ DK 2800 Lyngby, Denmark \\ smi@oersted.dtu.dk
}

\begin{abstract}
The performance of a previously developed algorithm for Radio Frequency Interference (RFI) detection and mitigation is experimentally evaluated. Results obtained from CoSMOS, an airborne campaign using a fully polarimetric L-band radiometer are analyzed for this purpose. Data is collected using two separate integration times, as a result of which sensitivity of the detection algorithm is measured. The impact of RFI on remotely sensed data over land and sea is also presented.
\end{abstract}

Keywords; microwave radiometer, radio frequency interference

\section{INTRODUCTION}

$\mathrm{R}$ adiometer signals received in the $\mathrm{L}$ and $\mathrm{C}$ band are particularly susceptible to man-made Radio Frequency Interference (RFI) [1,2]. RFI mitigation has become an important issue, as both ESA and NASA plan to launch satellite missions, SMOS and Aquarius respectively, responsible for measuring sea surface salinity (SSS) in the L-band. SMOS is also involved in determining soil moisture.

A second generation digital detection EMIRAD L-band radiometer was used in the CoSMOS campaign, an airborne mission in preparation of SMOS, to investigate sea surface brightness temperatures, as well as impact of RFI. The digital radiometer measures the higher order moments of the radiometer signals at two separate rates and uses a recently proposed kurtosis algorithm [3] to detect and mitigate RFI in post-processing.

Results from post-processing of data obtained from the airborne campaign, indicate the success of the detection algorithm in easily distinguishing natural thermal emissions from man-made sources near the signal noise floor. The kurtosis algorithm is also immune to any large natural variations in brightness temperature, such as coastal crossings.

The unique feature of the digital L-band radiometer of simultaneously measuring data at a faster rate, allows evaluation of the sensitivity of kurtosis to RFI brightness temperature contribution. Initial results confirm the ability of kurtosis algorithm to detect RFI near the $N E \Delta T$ level. RFI has a relatively higher percentage over land compared to the sea, probably due to urban areas.

\section{DETECTION ALGORITHM}

The radiometer generally detects natural thermal emissions as well as thermal noise of the hardware. These signals are random processes and hence the pdf of the amplitude of this signal has a Gaussian distribution. The kurtosis algorithm for RFI detection developed by Ruf et al. [3] takes advantage of the fact that almost all manmade sources would have a non-Gaussian distribution. RFI is detected by measuring the amount of deviation of the kurtosis parameter from normality.

The kurtosis algorithm measures the higher order central moments of the incoming radiometer signal to obtain the kurtosis. The $2^{\text {nd }}$ central moment (variance) obtained over a particular integration period is equivalent to the system noise temperature measured by the radiometer. The ratio of the $4^{\text {th }}$ central moment to the squared $2^{\text {nd }}$ central moment gives the kurtosis ratio, as shown in Equation $\mathbf{1}$.

$k=\frac{m_{4}}{m_{2}^{2}}$,

where,

$m_{i}=E\left[(x-E[x])^{i}\right]$

For natural thermal emissions, the distribution is Gaussian and the above ratio should ideally be equal to 3 . If the signal is corrupted by man-made RFI, the distribution should deviate from normality and thus $k$ should deviate from 3. Due to oversampling, such flagged data samples are then discarded and the clean samples are further integrated for scientific purposes. Since the algorithm is 
insensitive to change in the $2^{\text {nd }}$ moment, natural variations in the brightness temperature will not be falsely flagged as RFI.

It should be noted that the kurtosis parameter itself is affected by other factors such as digitization, bit-length, clipping, number of samples, correlation, filtering, nonstationarity etc. which add a certain noise-margin to it and have to be taken into account. These factors also cause the kurtosis ratio mean to be slightly different than 3 as shown in the bottom plot of Fig 2[4].

\section{CoSMOS CAMPAIGN}

The CoSMOS campaign had the EMIRAD radiometer with an aft and nadir-looking antenna installed in a Skyvan aircraft provided by the Helsinki University of Technology. The receiver is a fully polarimetric correlation radiometer with direct sampling operating in the 1400-1427 MHz region[5]. The signal obtained from the microwave front-end is fed into two $\mathrm{A} / \mathrm{D}$ converters operating at a sampling frequency of $139.4 \mathrm{MHz}$.

The digital front-end is responsible for integrating and dumping the $2^{\text {nd }}$ and $4^{\text {th }}$ moments onto a hard-disk. EMIRAD records the data at two separate integration periods simultaneously. The first integration period is of $8 \mathrm{~ms}$, which is known as the 'Slow' data. Similarly, $2^{\text {nd }}$ and $4^{\text {th }}$ moments are integrated over a much shorter period of $1.8 \mu \mathrm{s}$, and this data is appropriately termed as the 'Fast' data. EMIRAD employs a feed-back loop with the A/D converter which ensures that the first order moment (mean) of the signal remains zero, as a result the moments measured are effectively central moments.

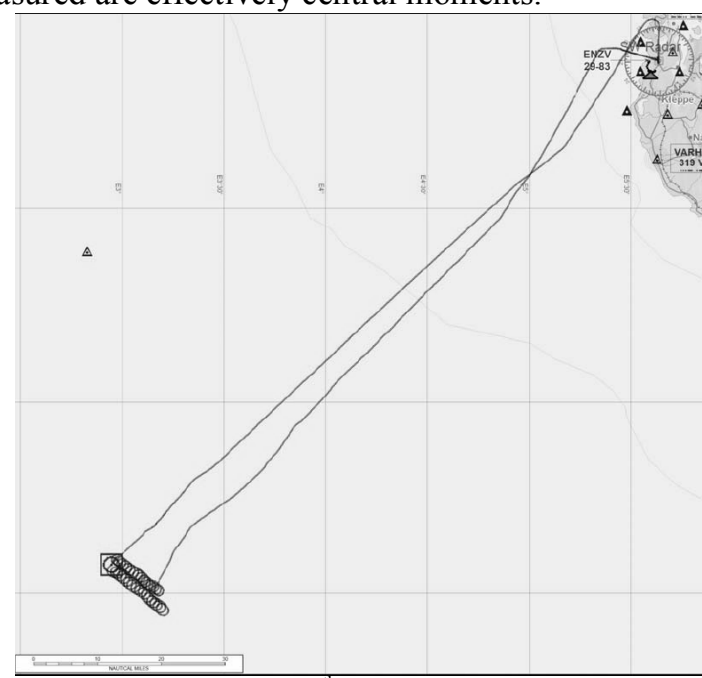

Fig. 1 Flight plan taken on the $19^{\text {th }}$ April, 2005 over the North-sea near the coast of Norway at 19:38 UTC

The airborne campaign took place over the North-sea near Norway. Data was processed from 13 separate days of flight, with each flight time approximately two to three hours long. The flight path taken is shown by Fig. 1.

\section{RESULTS OF RFI DETECTION}
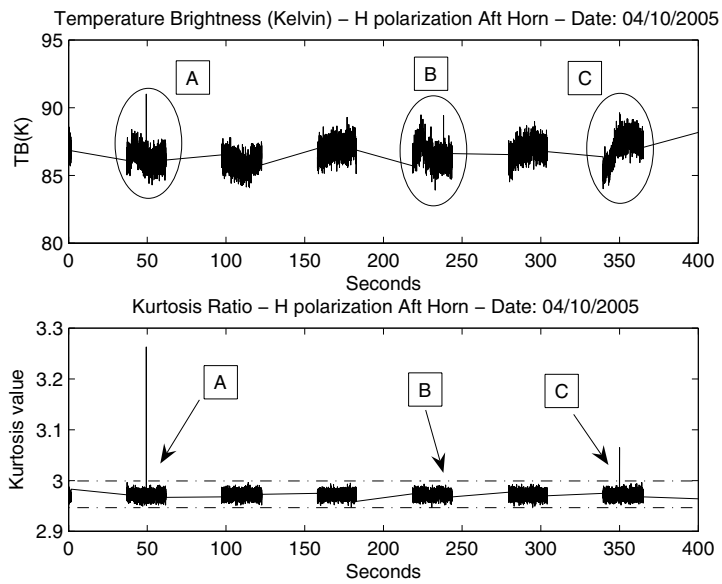

Fig. 2 (a) H-pol brightness temperature measured by the Aft horn. (b) Kurtosis plot corresponding to the brightness temperature plot. RFI-free data is contained within the dash-dot lines, points $\mathrm{A}$ and $\mathrm{C}$ represent two Slow data samples corrupted by RFI, point B represents an RFI free data sample.

As mentioned previously, the detection algorithm is able to flag RFI not apparently visible in the brightness temperature data. Fig 2 indicates 400 seconds of Slow data measured by the aft-horn, taken during the flight campaign, with the top plot indicating $\mathrm{Tb}$ (in Kelvin) and the bottom plot indicating the moment-ratio (kurtosis). The dashed lines in the bottom plot indicate the kurtosis threshold within which data is considered to be RFI free. The threshold is based on the noise-margin of the kurtosis statistic. The kurtosis mean is less than 3 due to external factors previously mentioned.

Points $\mathrm{A}$ and $\mathrm{C}$, shown in the plots are flagged as RFI in the data. Comparing them it is noticed from the top plot that there is a clear spike in the brightness temperature for the first point (A), whereas there is negligible difference in the temperature brightness of the second point (C) compared to its surroundings. This represents the ability of the kurtosis algorithm to detect man-made interference near the noise-margin of the radiometer data. This fact is later confirmed observing the Fast data, discussed in the next section. Point B is a counter example, where the Tb plot clearly indicates a spike and this might cause the data sample to be falsely flagged as RFI. On the other hand the bottom plot indicates the kurtosis completely within the set noise-margins, thus demonstrating that the sample is a part of a natural thermal emission. 

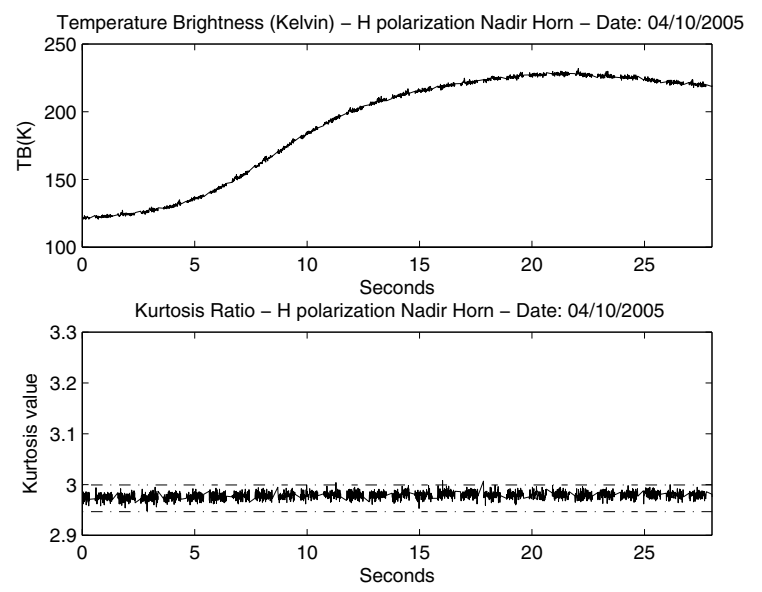

Fig. 3 (a) H-pol brightness temperature measured by the Nadir horn for a coastal crossing. (b) Kurtosis plot corresponding to the brightness temperature plot. RFI-free data is contained within the dash-dot lines.

An indication of the algorithm's robustness is its behavior under rapidly varying brightness temperature conditions. A portion of coastal crossing data from the sea to land is shown by Fig. 3. As seen in the lower plot, the kurtosis measurement is totally independent of the power, and hence it is an unbiased detector.

Flight campaigns over the North-sea indicated the actual amount of data corrupted by RFI when using the kurtosis method. Table 1, shows the percentage of horizontally polarized Slow data samples measured by the Nadir antenna corrupted by RFI, when over land and when over sea. The table gives a first indication towards the impact of RFI on remotely sensed data.

TABLE I.

Percentage of data corrupted by RFI over Land and Sea

\begin{tabular}{|c|c|c|}
\hline & \multicolumn{2}{|c|}{$\begin{array}{c}\text { RFI Percentage } \\
\text { (Nadir horn, H-pol) }\end{array}$} \\
\hline Date & Land & Sea \\
\hline 6.4 .05 & 9,327 & 0,314 \\
\hline 9.4 .05 & 7,131 & 0,062 \\
\hline 10.4 .05 & 6,397 & 0,046 \\
\hline 12.4 .05 & 1,328 & 0,043 \\
\hline 13.4 .05 & 5,854 & 0,868 \\
\hline 15.4 .05 & 2,047 & 0,059 \\
\hline 16.4 .05 & 19,523 & 3,373 \\
\hline 18.4 .05 & 0,307 & 0,055 \\
\hline 19.4 .05 & 6,847 & 2,388 \\
\hline 22.4 .05 & 23,360 & 43,947 \\
\hline 25.4 .05 & 4,382 & 0,062 \\
\hline 29.4 .05 & 41,698 & 53,340 \\
\hline 30.4 .05 & 1,223 & 0,441 \\
\hline
\end{tabular}

With the exception of two days, the $22^{\text {nd }}$ and $29^{\text {th }}$, data is relatively clean when over the sea surface. For most days RFI corruption is limited to less than one percent and doesn't exceed three percent on other days. As observed from the table, RFI corruption over land is nearly 2 to 100 times more than sea in terms of percentage. This is an expected result though, as the flight portion over land occurred near RFI intensive locations such as an airport.
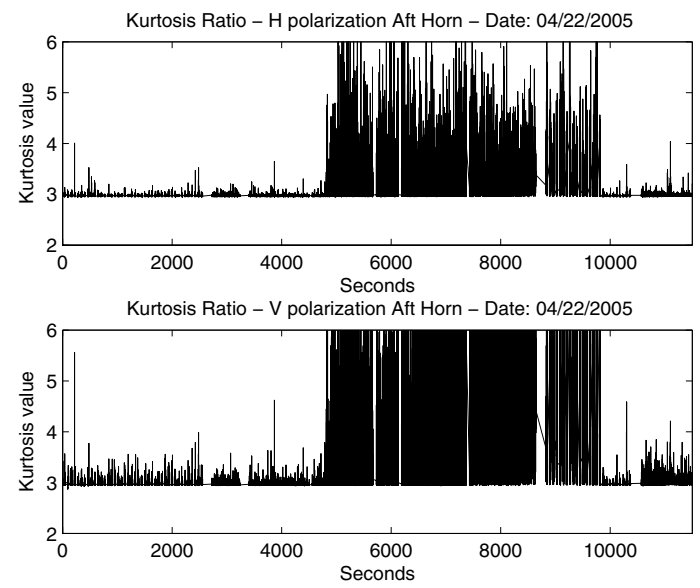

Fig. 4 Kurtosis plots of the $\mathrm{H}$ and $\mathrm{V}$ pol data measured on the $22^{\text {nd }}$ April, '05 indicating an unusually high amount of RFI over the sea (4500-10000 seconds)

Table 1 also shows that data taken on two days of the flight campaign, the $22^{\text {nd }}$ and $29^{\text {th }}$, have nearly 20 to $50 \%$ of the data corrupted by RFI. Fig. 4 shows the kurtosis plot of the H-pol and V-pol for one of the two days. Such an occurrence is highly unusual when compared to other days of data analysis. The reason for such an RFI burst over the sea surface is yet to be determined.

\section{PERFORMANCE OF KURTOSIS ALGORITHM}

The CoSMOS digital radiometer also measures and stores the second and fourth order moments with a much shorter integration time of $1.8 \mu \mathrm{s}$, and hence effectively faster sampling rate. RFI spikes are clearly visible from the $2^{\text {nd }}$ moment $(\mathrm{Tb})$ data. This offers an alternate method of detecting and mitigating RFI corrupted samples.
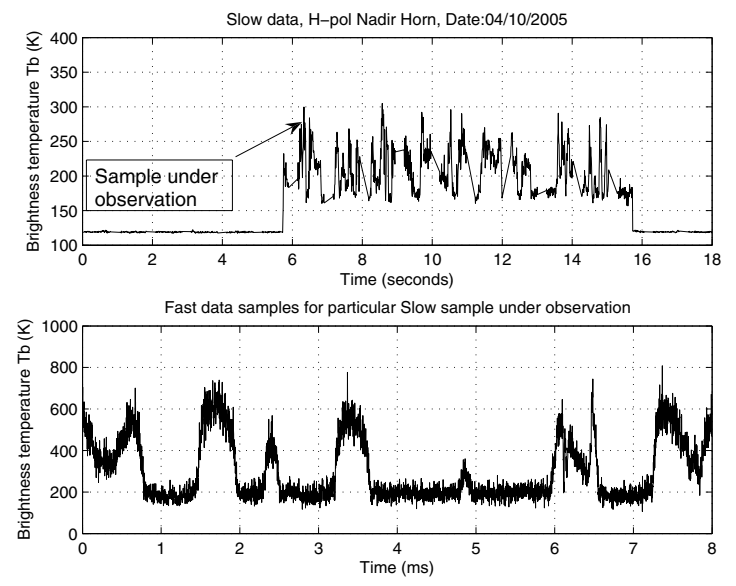

Fig. 5 The bottom plot shows Fast data samples corresponding to a particular Slow data sample indicated by the arrow in the top plot 
Such Fast data samples allow the ability to measure the amount of brightness temperature bias contributed by the RFI. This is done using a simple threshold algorithm. Variance of the $2^{\text {nd }}$ moment data is calculated from clean data observed during the calibration looks of the radiometer. Using this variance and mean of the observed data samples, a threshold is set. Any Fast sample with a value above this threshold is flagged as RFI, and the contributing brightness temperature is measured.

Each Slow data sample integrated over a period of $8 \mathrm{~ms}$, has approximately 4500 corresponding Fast data samples as shown above by Fig. 5. If any Slow data sample is flagged as RFI by kurtosis, by observing the associated Fast data samples it is possible to measure the exact amount of RFI contribution in terms of brightness temperature to which kurtosis is sensitive to.

Fig. 6 represents histograms of the amount of brightness temperature contributed by RFI for all days of the CoSMOS campaign, with the exception of the $22^{\text {nd }}$ and $29^{\text {th }}$, when integrated over a period of 1 second. The $N E \Delta T$ level for the digital radiometer is approximately $0.1 \mathrm{~K}$ when considering a 1 second integration period. As indicated by the figure, most of the RFI flagged by the kurtosis algorithm is below the $1 \mathrm{~K}$ region, with a higher concentration around the $N E \Delta T$ level. This confirms the better performance of the kurtosis algorithm when dealing with low-level RFI compared to traditional methods.
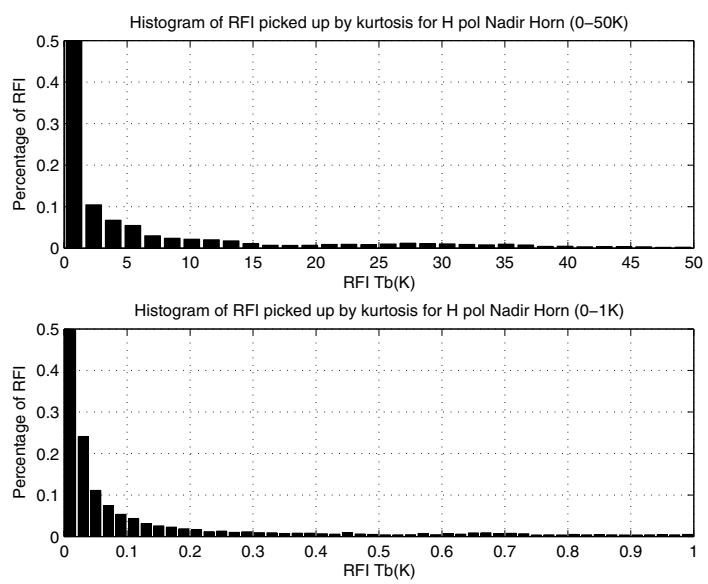

Fig. 6 Histograms indicating sensitivity of the kurtosis algorithm with respect to brightness temperature contributions from RFI when integrated over a period of 1 second. (a) From 0 to $50 \mathrm{~K}$ (b) From 0 to $1 \mathrm{~K}$

The above figure indicates that almost $50-60 \%$ of the RFI contribution is below the $1 \mathrm{~K}$ region, which can be ignored when considering applications such as soil moisture measurement, but has an impact up to $0.1 \mathrm{~K}$ when considering sea-surface salinity measurements. A major influence of RFI is observed around the 2 to $15 \mathrm{~K}$ region as indicated by the top plot. Such RFI is almost impossible to eliminate using conventional threshold algorithms. They would require very high sampling rates and low integration times such as the Fast data, which would strain data bandwidth resources. This is not an issue when dealing with kurtosis measurements, since Slow data measured at a longer integration time can easily flag RFI without consuming hardware or data bandwidth resources.

It should be noted that the kurtosis algorithm might not be able to flag all types of RFI operating around the $N E \Delta T$ region. Detection purely depends upon the type and distribution of the man-made interfering source[4]. Any man-made interference with a near-Gaussian distribution would be impossible to detect in this manner [3].

\section{CONCLUSIONS}

Performance of the RFI detection and mitigation algorithm has been presented using data obtained from an airborne second generation digital L-band radiometer system. Inclusion of the Fast data confirms the success of the kurtosis algorithm in flagging non-Gaussian sources. The kurtosis algorithm is independent of any brightness temperature variations. The detection algorithm is able to detect low-level RFI corrupted samples near the $N E \Delta T$ region of $0.1 \mathrm{~K}$ for a 1 second integration period. Results indicate that the presence of RFI over the sea-surface is less than $1 \%$, with RFI-intensive areas such as airports over land have nearly $5-7 \%$ or more of the data corrupted. In general, nearly $50 \%$ of the RFI contribution for most days of the CoSMOS flight campaign was below $1 \mathrm{~K}$. It should be noted that these results are specific to the North-sea south-west of the Norwegian coast, and will vary for different regions.

\section{ACKNOWLEDGEMENTS}

The authors would like to acknowledge their collaboration with the Helsinki University of Technology and ESTEC.

\section{REFERENCES}

[1] E.G.Njoku, P.Ashcroft, T.K.Chan, and L.Li, "Global survey and statistics of radio-frequency interference in AMSR-E land observations," IEEE Trans.Geoscience and Remote Sensing, vol. 43, no. 5, pp. 938947, May 2005.

[2] D.M.Le Vine and M.Haken, "RFI at L-band in synthetic aperture radiometers," in Proc. IGARSS, vol. 3, Tolouse, France, Jul. 21-25, 2003, pp. $1742-1744$

[3] C.S.Ruf, S.M.Gross, and S.Misra, "RFI Detection and Mitigation for Microwave Radiometry with an Agile Digital Detector," IEEE Trans. Geoscience and Remote Sensing, vol. 44, no. 3. pp. 694-706, March 2006.

[4] R.De Roo, S.Misra, C.S.Ruf, "Sensitivity of the kurtosis statistic as a detector of pulsed sinusoidal RFI," IEEE Trans. Geoscience and Remote Sensing, in press.

[5] N.Skou, S.S.Søbjærg, J.Balling, and S.S.Kristensen, "A Second Generation L-band Digital Radiometer for Sea Salinity Campaigns," Proceedings of IGARSS'06, August 2006, 4 p.. 